\title{
Fístula aortocava como complicación de aneurisma aórtico abdominal*
}

\author{
Dr. CARLO ZÚÑIGA G. ${ }^{1}$, Ints. JOSÉ RAMÓN RODRÍGUEZ A. ${ }^{2}$, PATRICIO CÁCERES G. ${ }^{2}$ \\ 1 Servicio de Cirugía Hospital Gustavo Fricke, Viña del Mar. \\ 2 Interno de Medicina, Universidad de Valparaíso, Valparaíso. \\ Chile.
}

\begin{abstract}
Aortocaval fistula as a complication of abdominal aortic aneurysm

Aortocaval fistula (ACF) is an infrequent complication of abdominal aortic aneurysm (AAA). Diagnosis is suspected by the presence of a continuous abdominal bruit and pulsatile abdominal mass, with variable signs of venous congestion and acute heart failure. Multislice computed tomography (MSCT) is useful in establishing the diagnosis, showing early enhancement of inferior vena cava and, in some cases, identifying the arteriovenous comunication. Surgical treatment is usually complex, with high morbidity and mortality rates. Endovascular treatment seems to be a promising alternative for the management of these patients. We report a case of ACF treated with open surgery and a literature review of this rare condition.
\end{abstract}

Key words: Aortic aneurysm, aortocaval fistula, vena cava.

\section{Resumen}

La fístula aortocava (FAC) es una complicación infrecuente del aneurisma aórtico abdominal (AAA). El diagnóstico se sospecha por la detección de un soplo continuo en el hemiabdomen inferior, asociado a masa abdominal pulsátil y signos variables de congestión venosa e insuficiencia cardíaca. La tomografía computada multicorte (TCM) permite confirmar el diagnóstico, evidenciando contraste de la vena cava inferior en fase arterial e identificando la zona anatómica de la comunicación arteriovenosa, en algunos casos. La reparación quirúrgica suele ser compleja, reportándose una alta morbi-mortalidad asociada. El uso de técnicas endovasculares pareciera mejorar el pronóstico de esta entidad. Reportamos un caso de FAC tratado mediante cirugía abierta. Se incluye una revisión de la literatura respecto a esta rara condición.

Palabras clave: Aneurisma aórtico abdominal, fístula aortocava, vena cava inferior.

\section{Introducción}

La fístula aortocava (FAC) es una complicación infrecuente del Aneurisma Aórtico Abdominal (AAA), cuya incidencia varía entre un 1 a $6 \%$ de los aneurismas complicados ${ }^{1-4}$.
La presentación clásica comprende la tríada de dolor abdominal, masa abdominal pulsátil y soplo continuo en hemiabdomen inferior. Ocasionalmente, la presencia de signos de congestión venosa e insuficiencia cardíaca de inicio agudo, pueden sugerir el diagnóstico ${ }^{4-7}$.

*Recibido el 8 de octubre de 2010 y aceptado para publicación el 15 de enero de 2011.

Correspondencia: Dr. Carlo Zúñiga G.

Álvarez 1532, Viña del Mar, Chile. Fax: (032) 2652343, (032) 2652346.

cfzuniga@yahoo.com 
Ante la sospecha clínica, es fundamental contar con imágenes de alta resolución, en especial la tomografía computada multicorte (TCM), la cual permite confirmar el diagnóstico y planificar la estrategia quirúrgica en pacientes estables ${ }^{8,9}$.

Reportamos un caso de FAC espontánea secundario a aneurisma aortoilíaco complicado, intervenido de urgencia a través de abordaje clásico transperitoneal; se presenta una revisión de la literatura respecto a esta entidad.

\section{Caso clínico}

Paciente de sexo masculino de 68 años de edad, con antecedentes de hipertensión arterial esencial y tabaquismo activo. Consulta en la Unidad de Emergencias del Hospital Gustavo Fricke, por cuadro de tres días de dolor en hipogastrio y fosa ilíaca izquierda, de inicio súbito. Al examen se encontraba normotenso, con taquicardia leve y palidez de piel y mucosas. Se detectó masa pulsátil supraumbilical sensible y soplo abdominal continuo en hipogastrio. Se efectuó una ecotomografía abdominal que reveló la presencia de un gran AAA. Posteriormente, se realizó TCM (Siemens Somatom Emotion de 16 canales) que mostró un extenso AAA infrarrenal, de 7,7 centímetros de diámetro antero-posterior máximo, con presencia de dilatación aneurismática de ambas arterias ilíacas comunes y comunicación a la vena cava inferior (VCI), por sobre la bifurcación de las venas ilíacas comunes (Figuras 1 y 2). Mediante reconstrucción tridimensional se evidenció presencia de la FAC a nivel de la cuarta vértebra lumbar (Figura 3).

Se procedió a efectuar reparación abierta por vía transperitoneal, evidenciándose frémito en la VCI.

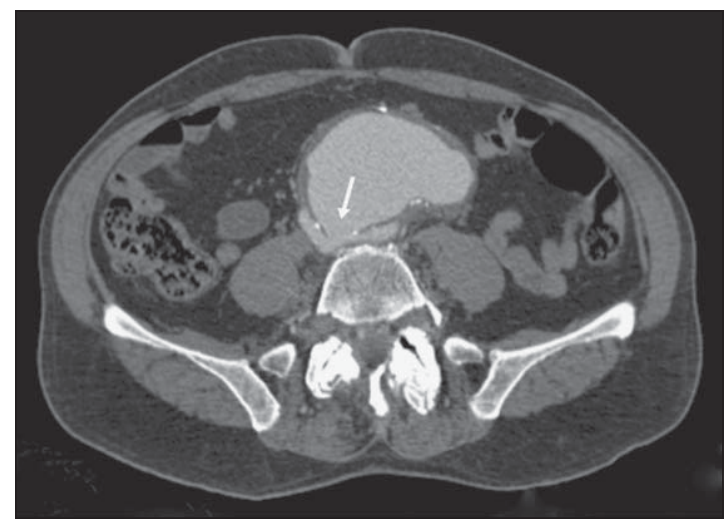

Figura 1. TCM con contraste. Comunicación de la aorta con la VCI (flecha), sobre la bifurcación de las venas ilíacas comunes y contraste precoz de VCI.
Una vez obtenido control vascular, se abrió el saco aneurismático y se suturó el defecto, desde el lumen aórtico hacia la VCI, mediante sutura continua de Prolene. A continuación, se realizó interposición de prótesis bifurcada de Dacron precoagulado, de 16 x $8 \mathrm{~mm}$, entre la aorta infrarrenal y ambas arterias

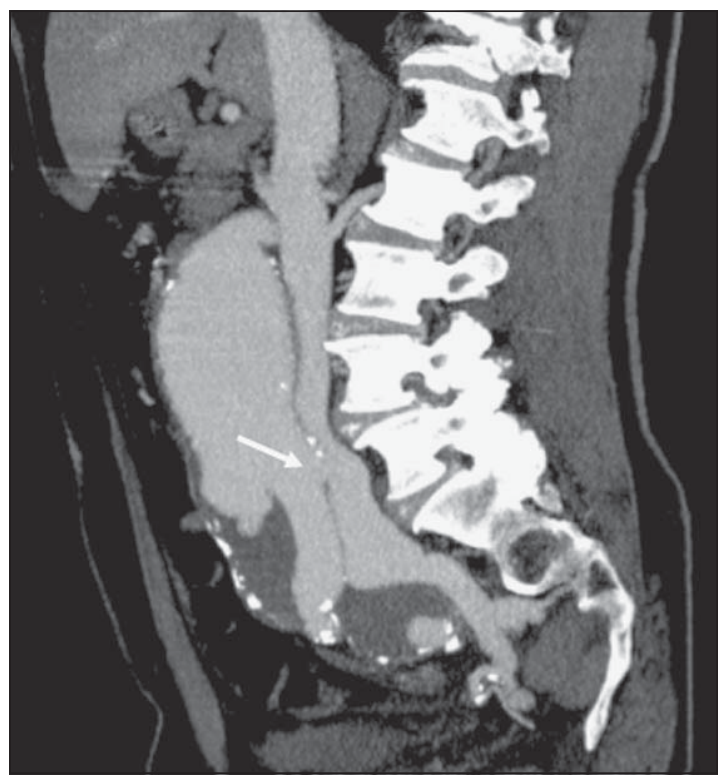

Figura 2. Reconstrucción sagital. Destaca comunicación entre AAA y VCI (flecha), con llene retrógrado de vena renal izquierda.

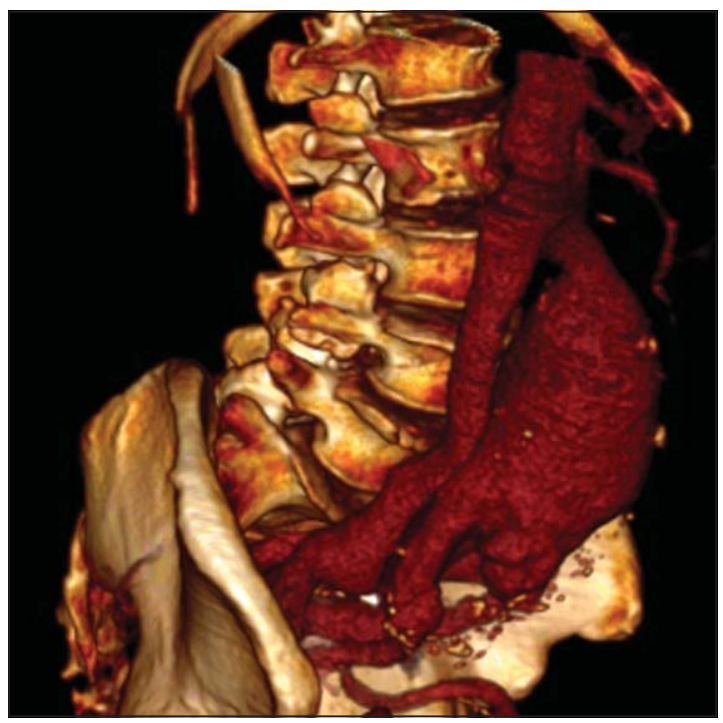

Figura 3. Reconstrucción tridimensional. Se evidencia extenso AAA con contraste precoz de VCI. 
ilíacas externas. Ambas arterias hipogástricas fueron ligadas y se reimplantó la arteria mesentérica inferior en forma termino lateral a la rama ilíaca izquierda del bypass, para evitar la isquemia del colon izquierdo.

Durante el post operatorio, el paciente presentó isquemia aguda de la pierna derecha, secundaria a disección de la arteria ilíaca externa, distal a la anastomosis. Se realizó trombectomía y corrección del defecto técnico, sin embargo, el paciente evolucionó en forma catastrófica con rabdomiolísis, insuficiencia renal aguda y finalmente falla orgánica múltiple, falleciendo 36 horas luego de la intervención inicial.

\section{Discusión}

La FAC espontánea fue descrita inicialmente por Syme en $1831^{10}$. En más del $80 \%$ de los casos, la comunicación arteriovenosa es provocada por la ruptura de un aneurisma aterosclerótíco hacia el sistema venoso ${ }^{5}$. Un porcentaje importante se presenta en el contexto de aneurismas traumáticos, especialmente luego de lesiones penetrantes abdominales ${ }^{11}$. Ocasionalmente se han descrito casos como complicación de cirugía de hernia lumbar ${ }^{5}$, FAC secundarias a aneurismas sifilíticos y aneurismas micóticos ${ }^{10} \mathrm{O}$ asociadas a entidades poco frecuentes como síndrome de Ehler Danlos ${ }^{12}$, síndrome de $\operatorname{Marfan}^{13}$ y arteritis de Takayasu ${ }^{14}$.

La presentación clínica está relacionada con el tamaño y localización de la comunicación arteriovenosa $^{8}$. La tríada clásica comprende la presencia de dolor abdominal, masa pulsátil palpable y soplo abdominal continuo, como fue el caso del paciente descrito ${ }^{5,6}$. El dolor se encuentra presente en aproximadamente la mitad de los casos. Habitualmente se localiza en hipogastrio o región lumbar y puede irradiarse a la ingle o muslos ${ }^{4,7}$. Más de un $85 \%$ de los pacientes presentará masa abdominal palpable y en cerca del $70 \%$ de los casos es posible objetivar la presencia de soplo abdominal ${ }^{4}$. Otras formas de presentación son consecuencia del cortocircuito izquierda-derecha. En FAC de gran tamaño es posible evidenciar signos de falla cardíaca de alto flujo, edema pulmonar, congestión venosa central, hepatomegalia y ascitis ${ }^{4,7}$. Si el cortocircuito está dirigido hacia el territorio venoso periférico, la presión venosa central puede estar normal, con signos de hipertensión venosa regional, produciendo edema, venas varicosas pulsátiles y cianosis de extremidades inferiores ${ }^{6,15}$. Adicionalmente, algunos pacientes pueden cursar con rectorragia, priapismo o hematuria, secundaria a congestión venosa de la pared vesical ${ }^{16,17}$. La presencia de falla renal no es infrecuente, debido a hipoperfusión e hipertensión venosa renal ${ }^{18}$.

La importancia del diagnóstico preoperatorio radica en favorecer una adecuada planificación anestésica y quirúrgica. Esto permtiría al cirujano poner especial enfásis en reducir el sangrado intraoperatorio y prevenir el paso de aire, trombos o fragmentos ateroscleróticos desde el aneurisma a la $\mathrm{VCI}^{6-9}$. Sin embargo, en cerca del 50\% de los casos, los signos y síntomas clásicos suelen estar ausentes, dificultando el diagnóstico ${ }^{5,6}$. En pacientes estables, la ecotomografía doppler abdominal permite confirmar el diagnóstico en algunos $\operatorname{casos}^{19}$. En aquellos pacientes en que la ecotomografía no es concluyente, la TCM con contraste es el examen de elección. Algunos signos que sugieren la presencia de una FAC son la pérdida de la interfase grasa entre VCI y aorta, pasaje precoz de contraste a la VCI y la visualización de la fistula ${ }^{17,20,21}$. Aun cuando no es considerado un procedimiento esencial, en casos seleccionados la arteriografía puede confirmar el diagnóstico, además de entregar información respecto a la ubicación y tamaño de la fistula ${ }^{7}$.

El tratamiento quirúrgico clásico, está dirigido a la obtención del control vascular del aneurisma, seguido por cierre directo de la comunicación arteriovenosa desde el saco aneurismático ${ }^{21}$. Si la estructura de la arteria ilíaca se encuentra conservada, el uso de parches protésicos puede ser útil para la reconstrucción del aneurisma ${ }^{19}$, de lo contrario, se debe recurrir a la confección de un bypass aortobifemoral $^{8}$. La mortalidad perioperatoria es variable, con cifras que van de 0 a $67 \% \%^{4,6,16,19,21}$. Se ha descrito que en FAC con AAA rotos hacia el retroperitoneo o hacia la cavidad abdominal, la mortalidad duplicaría a aquellos casos de FAC no complicada ${ }^{21}$.

El manejo endovascular ofrece una alternativa terapéutica atractiva respecto a la cirugía abierta, con resultados satisfactorios descritos en al menos seis casos $^{22-27}$. No obstante, el número de pacientes tratados por esta vía sigue siendo escaso y el seguimiento a largo plazo es insuficiente. Se ha teorizado que la comunicación persistente entre VCI y el saco aórtico, podría facilitar el desarrollo de un endoleak tipo II de alto flujo, sin embargo, la evidencia disponible aún no es concluyente al respecto ${ }^{24,27,28}$.

En conclusión, la FAC es una complicación poco frecuente, cuyo diagnóstico depende de un alto índice de sospecha clínica, asociado al uso de imagenología no invasiva. El éxito del tratamiento está condicionado por el diagnóstico precoz y la resolución quirúrgica expedita. Pese a la evolución desfavorable del caso descrito, se pretende sensibilizar al clínico respecto a las manifestaciones de esta entidad, su diagnóstico y las alternativas terapéuticas disponibles en la actualidad. 


\section{Referencias}

1. Baker WH, Sharzer LA, Ehrenhaft JL. Aortocaval fistula as a complication of abdominal aortic aneurysms. Surgery 1972;72:933-8.

2. Lanne T, Bergqvist D. Aortocaval fistulas associated with ruptured abdominal aortic aneurysms. Eur J Surg. 1992;158:457-65.

3. Schmidt R, Bruns C, Walter M, Erasmi H. Aorto-caval fistula-an uncommon complication of infrarenal aortic aneurysms. Thorac Cardiovasc Surg. 1994;42:208-11.

4. Bednarkiewicz M, Pretre R, Kalangos A, Khatchatourian G, Bruschweiler I, Faidutti B. Aortocaval fistula associated with abdominal aortic aneurysm: a diagnostic challenge. Ann Vasc Surg. 1997;11:464-6.

5. Alexander JJ, Imbembo AL. Aorta-vena cava fistula. Surgery 1989;105:1-12.

6. Fenster MS, Dent JM, Tribble C, Angle JF, Sarembock IJ, Komada M, et al. Aortocaval fistula complicating abdominal aortic aneurysm: case report and literature review. Cathet Cardiovasc Diagn. 1996;38:75-9.

7. Potyk DK, Guthrie CR. Spontaneous aortocaval fistula. Ann Emerg Med. 1995;25:424-7.

8. Iriz E, Ozdogan ME, Erer D, Uslu M, Koksal P, Kurtipek O. A giant aortocaval fistula due to abdominal aortic aneurysm. Int J Cardiol. 2006;112:e78-80.

9. Tsolakis JA, Papadoulas S, Kakkos SK, Skroubis G, Siablis D, Androulakis JA. Aortocaval fistula in ruptured aneurysms. Eur J Vasc Endovasc Sur 1999;17:390-3.

10. Syme, J. Case of spontaneous varicose aneurysm. Edinb Med Surg J. 1831;36:1045.

11. Davidovic LB, Kostic DM, Cvetkovic SD, Jakovljevic NS, Stojanov PL, Kacar AS, et al. Aorto-caval fistulas. Cardiovasc Surg. 2002;10:555-60.

12. Lynch HT, Larsen AL, Wilson R, Magnuson CL. Ehlers-Danlos syndrome and "congenital" arteriovenous fistulae. A clinicopathologic study of a family. JAMA 1965;194:1011-4.

13. Hardin CA. Ruptured abdominal aneurysm occurring in Marfan's syndrome; attempted repair with the use of a nylon prosthesis. N Engl J Med. 1959;260 821-2.

14. Gronemeyer PS, De Mello DE. Takayasu's disease with aneurysm of right common iliac artery and iliocaval fistula in a young infant: case report and review of the literature. Pediatrics 1982;69:626-31.

15. Karanikis P, Korantzopoulos P, Theodorou A, Tsimoyiannis J, Siogas K. Leg oedema and exertional dyspnea due to aortocaval fistula complicating an abdominal aortic aneurysm. Int J Cardiol. 2004;94:335-7.

16. Brewster DC, Ottinger LW, Darling RC. Hematuria as a sign of aorto-caval fistula. Ann Surg. 1977;186:76671.

17. Hervas V, Esteban JM, García-Ferrer L. Aortocaval Fistula Presenting with Hematuria and Renal Failure. EJVES 2007;14:33-5.

18. Calligaro KD, Savarese RP, De Laurentis DA. Unusual aspects of aortovenous fistulas associated with ruptured abdominal aortic aneurysms. J Vasc Surg. 1990;12:58690.

19. Lin PH, Bush RL, Lumsden AB. Aortocaval fistula. J Vasc Surg. 2004;39:266.

20. Rosenthal D, Atkins CP, Jerrius HS, Clark MD, Matsuura JH. Diagnosis of aortocaval fistula by computed tomography. Ann Vasc Surg. 1998;12:86-7.

21. Maeda H, Umezawa H, Goshima M, Hattori T, Nakamura T, Nishii T, et al. Surgery for ruptured abdominal aortic aneurysm with an aortocaval and iliac vein fistula. Surg Today 2007;37:445-8.

22. Umscheid T, Stelter WJ. Endovascular treatment of an aortic aneurysm ruptured into the inferior vena cava. J Endovasc Ther. 2000;7:31-5.

23. Lau LL, O’reilly MJ, Johnston LC, Lee B. Endovascular stent-graft repair of primary aortocaval fistula with an abdominal aortoiliac aneurysm. J Vasc Surg. 2001;33: 425-8.

24. Vetrhus M, McWilliams R, Tan CK, Brennan J, GillingSmith G, Harris PL. Endovascular repair of abdominal aortic aneurysms with aortocaval fistula. Eur J Vasc Endovasc Surg. 2005;30:640-3.

25. Kopp R, Weidenhagen R, Hoffmann R, Waggershauser $\mathrm{T}$, Meimarakis G, Andrassy J, et al. Immediate endovascular treatment of an aortoiliac aneurysm ruptured into the inferior vena cava. Ann Vasc Surg. 2006;20:525-8.

26. León LR Jr, Arslan B, Ley E, Labropoulos N. Endovascular therapy of spontaneous aortocaval fistulae associated with abdominal aortic aneurysms. Vascular 2007; 15:35-40.

27. Guzzardi G, Fossaceca R, Divenuto I, Musiani A, Brustia P, Carriero A. Endovascular treatment of ruptured abdominal aortic aneurysm with aortocaval fistula. Cardiovasc Intervent Radiol. 2010;33:853-6.

28. Pevec WC, Lee ES, Lamba R. Symptomatic, acute aortocaval fistula complicating an infrarenal aortic aneurysm. J Vasc Surg. 2010;51:475. 